\title{
Vector biology prospects in dengue research
}

\author{
Louis Lambrechts ${ }^{1 /+}$, Anna-Bella Failloux ${ }^{2}$ \\ 1Department of Infection and Epidemiology, Insects and Infectious Diseases Unit, Associated Research Unit 3012, National Center for \\ Scientific Research, Paris, France ${ }^{2}$ Laboratory of Arboviruses and Insect Vectors, Department of Virology, Pasteur Institute, Paris, France \\ We argue that using more natural blood feeding methods to study mosquito vector competence for dengue viruses \\ and exploring the effect of viral infection on other mosquito life-history traits that influence vectorial capacity will \\ significantly advance our understanding of dengue epidemiology.
}

Key words: dengue - Aedes aegypti - Aedes albopictus - vector competence

Dengue disease is a growing public health burden in tropical and subtropical regions throughout the world. With at least two-fifths of the world's population living in areas that are vulnerable to dengue epidemics and 50-100 million infections each year, dengue is the most prevalent arboviral disease of humans worldwide (Guzman et al. 2010). Recent results from a vaccine trial showing only partial protection (Sabchareon et al. 2012) suggest that dengue will continue to impact global public health for the years to come. Dengue fever and its severe form, dengue haemorrhagic fever, are caused by RNA viruses of the genus Flavivirus that are transmitted by mosquitoes of the genus Aedes. At present, controlling mosquito vector populations is the primary dengue prevention strategy. Studying the biology of mosquito vectors and their role in the transmission of dengue viruses is an active field of research that has generated important knowledge for our understanding of dengue epidemiology. Failure to prevent the continued global expansion of dengue, however, suggests that this understanding is insufficient. In this article, we review some of the limitations that we think have hampered dengue research in the field of vector biology. In particular, we advocate bridging the gap between field conditions of transmission and laboratory models of vector-virus interactions. We highlight two major research avenues that will further advance our understanding of vectorial transmission of dengue viruses and ultimately help to prevent or mitigate this debilitating disease: (i) designing experimental approaches that reproduce as closely as possible natural infections of mosquitoes and (ii) extending research on vector-virus interactions beyond vector competence to encompass other mosquito life-history traits that determine vectorial capacity.

Vector competence is defined as the intrinsic ability of an arthropod to become infected and subsequently transmit a pathogen (Kramer \& Ebel 2003). Arthropods differ significantly in their vector competence for a given arthropod-borne virus (arbovirus) both between and within vector species. Presently, the mosquito Aedes aegypti is

\footnotetext{
+ Corresponding author: louis.lambrechts@pasteur.fr Received 26 November 2012

Accepted 28 November 2012
}

the principal dengue vector (Lambrechts et al. 2010). Due to its close association with humans, it has played a major role in worldwide dengue emergence in the last halfcentury. Although Aedes albopictus has been incriminated in small-scale dengue epidemics, it is considered a minor dengue vector compared to Ae. aegypti because of its lower vector competence and reduced aptitude to bite humans (Lambrechts et al. 2010). Nevertheless, the potential role of Ae. albopictus as a dengue vector has become a major concern in dengue-free temperate regions where this mosquito has been established in the absence of Ae. aegypti. Whereas Ae. aegypti was, not so long ago, considered the primary vector of chikungunya virus, a single viral mutation conferred enhanced transmission by Ae. albopictus and was subsequently selected in places where Ae. albopictus is the dominant vector species (de Lamballerie et al. 2008). This invasive mosquito has been incriminated as the principal vector in several recent chikungunya outbreaks in various locations around the world, including Italy (Angelini et al. 2008).

Historical methods of determining vector competence have had important experimental constraints that limit our ability to extrapolate to natural transmission and to understand the significance of data from epidemiological studies with humans. In particular, this research has, almost exclusively, been restricted to artificial infectious blood meals, largely due to ethical and logistical concerns associated with studies of naturally infected human subjects and the lack of a relevant animal model. Artificial infectious blood meals are generally composed of cultured virus mixed with animal blood presented to mosquitoes across a skin-simulating membrane. These methods are known to have a direct impact on virus-vector interactions in at least three important ways. First, common use of defibrinated blood in the infectious artificial blood meals can alter the distribution of virus within the mosquito midgut and result in a systematic underestimation of infection rates in experimentally exposed mosquitoes (Weaver et al. 1993). Second, passage in cell culture can select for viral mutations that are not maintained in nature and may affect the ability of the virus to replicate in a different cell type (Weaver et al. 1999). Third, the blood of a viraemic human may contain several immune factors (e.g. rising antibody titres) and other blood components that can impact vector com- 
petence, but are not present in an artificial infectious blood meal. Variation in Ae. aegypti vector competence for dengue viruses has been extensively characterised using artificially-exposed mosquitoes challenged with cell-cultured dengue viruses, but presently there is little data available to calibrate this knowledge to what is expected during natural human-to-mosquito transmission.

There have been a few, notable exceptions to these standard methods. In their classic studies during the 1920s and 1930s, Siler et al. (1926) and Simmons et al. (1931) were able to demonstrate, through human-tomosquito-to-human dengue virus transmission experiments, that the intrinsic (human host) incubation period of the virus was on average six days, but could be as long as 12 days. The human infectious period typically lasted four to five days and Ae. aegypti females infected after biting viraemic people were able to transmit the virus by bite to a second person after a minimum of 11 days of extrinsic (mosquito host) incubation. Half a century later, Gubler et al. (1978) examined transmission of dengue virus from people to mosquitoes by allowing laboratoryreared mosquitoes to feed on the arm of dengue-infected patients in Tonga. In this latter study, only one out of six participants was infectious to mosquitoes.

New studies based on direct exposure of people to mosquitoes are needed to document the extent of variation in human dengue infectiousness to mosquitoes and to elucidate the underlying host factors. Although we expect infectiousness to be positively correlated with viraemia, the precise shape of the relationship (between viraemia titre and the proportion of transmitting mosquitoes) and the influence of co-factors are currently unknown. While direct feeding methods have not been used extensively in arbovirology, there is a substantial body of work in malariology that is based on more natural feeding techniques. Several studies have explored interactions between Plasmodium parasites and anopheline mosquitoes by feeding laboratory-reared mosquitoes on people with active malaria infections, alone, or in comparison with artificial feeding methods, providing valuable experimental models (Bonnet et al. 2000, Awono-Ambene et al. 2001, Diallo et al. 2008).

In addition to vector competence, several other entomological parameters contribute to vectorial capacity, which reflects the overall contribution of the vector population to pathogen transmission (Kramer \& Ebel 2003). They include the vector biting rate, vector density, vector survival and the duration of the virus extrinsic incubation period. To date, research on the interactions between dengue viruses and their mosquito vectors has primarily focused on vector competence. The most obvious reason is that other parameters, most notably the biting rate, are difficult to measure in an experimental setting. Howev$\mathrm{er}$, the relative contribution of variation in vector competence to changes in vectorial capacity is small compared to, for example, variation in biting rate or survival. This is because, mathematically, vector competence is linearly related to vectorial capacity whereas biting rate and survival influence vectorial capacity according to a power function (Kramer \& Ebel 2003). For example, the biting rate is elevated to the power 2 in the equation of vectorial capacity because a mosquito must bite a human twice for virus transmission to occur (the 1st time to acquire the virus, the 2 nd to deliver it). An important gap in our knowledge is to understand how these mosquito life-history traits, biting rate and survival, are affected by dengue virus infection.

Like most arboviruses, dengue viruses establish infection in many different mosquito organs, including the salivary glands and the central nervous system. The salivary glands store the infectious viral particles that are released into the saliva and inoculated when the mosquito female probes the host skin. Both the probing time and the time required to acquire a blood meal may be longer in dengueinfected mosquitoes compared to uninfected ones (Platt et al. 1997). This could be a mechanical consequence of the virus disturbing the physiology of the salivary glands or an indirect behavioural modification induced by viral invasion of the brain tissue. A recent study showed that Ae. aegypti odorant-binding protein genes involved in host-seeking or probing behaviour are modulated by dengue virus infection (Sim et al. 2012). From an evolutionary standpoint, this change in mosquito behaviour may provide a selective advantage to the virus and may be the result of natural selection. Indeed, an extended duration of blood feeding increases the risk of interruption by the host, which in turn increases the probability that the infected mosquito feeds on additional host(s), thus creating more opportunities for virus transmission.

Viral infection induces manifold effects in mosquitoes that may cause fitness costs resulting from reallocation of energy resources towards viral replication to the detriment of life-history traits such as survival. Across different vector-virus systems, the effect of arboviral infection on mosquito survival is modest, but significantly negative (Lambrechts \& Scott 2009). In a striking example, Ae. aegypti females infected with dengue virus died, on average, twice as fast as uninfected females (Macielde-Freitas et al. 2011). Vector longevity was negatively associated with viral load at death, suggesting that the intensity of viral replication was directly related to the reduction in survival. In addition, the longevity of mosquitoes that were exposed but cleared the infection was reduced compared to infected mosquitoes, indicating that resistance mechanisms may be more costly than tolerance mechanisms. Presumably, the negative effect of viral replication on vector survival does not result from a direct pathogenic effect, but rather from an indirect effect due to metabolism disturbance. Although reduced vector survival negatively impacts the probability of virus transmission, evolutionary theory predicts that the overall outcome can be advantageous to the virus if increased virulence (the reduction of host fitness due to infection) also confers enhanced horizontal transmission (Lambrechts \& Scott 2009). For instance, increased frequency of vector-host contacts resulting from the manipulation of blood feeding behaviour in heavily infected mosquitoes may counterbalance the negative effects on survival.

Perhaps to a lesser extent, dengue infection is also likely to alter other mosquito life-history traits than survival and biting rate. Infected mosquitoes may allocate more resources to reproduction as a compensa- 
tory mechanism to maintain fitness in spite of a shorter lifespan. Alternatively, the energy diverted by viral replication may result in an additional fitness cost in infected mosquitoes in the form of reduced fecundity. A negative impact on egg production was observed in dengue-infected Ae. aegypti (Maciel-de-Freitas et al. 2011). Dengue infection may also alter behavioural traits that are not directly related to blood feeding such as locomotor activity (Lima-Camara et al. 2011). Increased locomotor activity in dengue-infected mosquitoes could potentially increase the chances to find a host (Luz et al. 2011). Alternatively, dengue-infected mosquitoes could be less agile in their movements and therefore become more vulnerable to predation as was demonstrated for insecticide resistant mosquitoes (Berticat et al. 2004).

To date, most studies on dengue virus transmission by mosquitoes have focused on the outcome of direct interactions between the vector and the virus that contribute to determine vector competence. The majority of these studies have relied on laboratory systems that use artificial infectious blood meals. We suggest that addressing both of these limitations in future studies will provide new insights into dengue epidemiology. Using more natural feeding techniques is necessary to determine the influence of vertebrate host factors on infectiousness to mosquitoes and to evaluate the relative contribution of infected people to virus transmission. Wherever possible, vector competence assays should be performed using field-derived mosquitoes from the local population that are exposed to the blood of viraemic people by directly feeding on them. Indirect feeding on viraemic blood drawn from a dengue-infected person via an artificial feeder is an alternative when direct feeding is not possible or deemed unethical. Finally, vector competence is only one of several entomological parameters underlying vectorial capacity and certainly not necessarily the most influential. The effects of dengue virus infection on other mosquito life-history traits that influence virus transmission need to be explored in more detail. Understanding the relationships between vector fitness and arboviral infection has important implications for predicting the evolution of vector-virus relationships and their role in the emergence and maintenance of arboviruses.

\section{ACKNOWLEDGEMENTS}

To Richard Paul, for helpful comments on the paper.

\section{REFERENCES}

Angelini P, Macini P, Finarelli AC, Pol C, Venturelli C, Bellini R, Dottori M 2008. Chikungunya epidemic outbreak in Emilia-Romagna Italy during summer 2007. Parassitologia 50: 97-98.

Awono-Ambene HP, Diawara L, Robert V 2001. Comparison of direct and membrane feeding methods to infect Anopheles arabiensis with Plasmodium falciparum. Am J Trop Med Hyg 64: 32-34.

Berticat C, Duron O, Heyse D, Raymond M 2004. Insecticide resistance genes confer a predation cost on mosquitoes Culex pipiens. Genet Res 83: 189-196.

Bonnet S, Gouagna C, Safeukui I, Meunier JY, Boudin C 2000. Comparison of artificial membrane feeding with direct skin feeding to estimate infectiousness of Plasmodium falciparum gametocyte carriers to mosquitoes. Trans R Soc Trop Med Hyg 94: 103-106. de Lamballerie X, Leroy E, Charrel RN, Ttsetsarkin K, Higgs S, Gould EA 2008. Chikungunya virus adapts to tiger mosquito via evolutionary convergence: a sign of things to come? Virol J 5: 33.

Diallo M, Toure AM, Traore SF, Niare O, Kassambara L, Konare A, Coulibaly M, Bagayogo M, Beier JC, Sakai RK, Toure YT, Doumbo OK 2008. Evaluation and optimization of membrane feeding compared to direct feeding as an assay for infectivity. Malar J 7: 248.

Gubler DJ, Reed D, Rosen L, Hitchcock Jr JR 1978. Epidemiologic, clinical and virologic observations on dengue in the Kingdom of Tonga. Am J Trop Med Hyg 27: 581-589.

Guzman MG, Halstead SB, Artsob H, Buchy P, Farrar J, Gubler DJ, Hunsperger E, Kroeger A, Margolis HS, Martinez E, Nathan MB, Pelegrino JL, Simmons C, Yoksan S, Peeling RW 2010. Dengue: a continuing global threat. Nat Rev Microbiol 8 (Suppl.): S7-S16.

Kramer LD, Ebel GD 2003. Dynamics of flavivirus infection in mosquitoes. Adv Virus Res 60: 187-232.

Lambrechts L, Scott TW 2009. Mode of transmission and the evolution of arbovirus virulence in mosquito vectors. Proc Biol Sci 276: 1369-1378.

Lambrechts L, Scott TW, Gubler DJ 2010. Consequences of the expanding global distribution of Aedes albopictus for dengue virus transmission. PLoS Negl Trop Dis 4: e646.

Lima-Camara TN, Bruno RV, Luz PM, Castro MG, Lourenço-deOliveira R, Sorgine MH, Peixoto AA 2011. Dengue infection increases the locomotor activity of Aedes aegypti females. PLoS ONE 6: e17690.

Luz PM, Lima-Camara TN, Bruno RV, de Castro MG, Sorgine MHF, Lourenço-de-Oliveira R, Peixoto AA 2011. Potential impact of a presumed increase in the biting activity of dengue-virus-infected Aedes aegypti (Diptera: Culicidae) females on virus transmission dynamics. Mem Inst Oswaldo Cruz 106: 755-758.

Maciel-de-Freitas R, Koella JC, Lourenço-de-Oliveira R 2011. Lower survival rate, longevity and fecundity of Aedes aegypti (Diptera: Culicidae) females orally challenged with dengue virus serotype 2. Trans R Soc Trop Med Hyg 105: 452-458.

Platt KB, Linthicum KJ, Myint KS, Innis BL, Lerdthusnee K, Vaughn DW 1997. Impact of dengue virus infection on feeding behavior of Aedes aegypti. Am J Trop Med Hyg 57: 119-125.

Sabchareon A, Wallace D, Sirivichayakul C, Limkittikul K, Chanthavanich P, Suvannadabba S, Jiwariyavej V, Dulyachai W, Pengsaa K, Wartel TA, Moureau A, Saville M, Bouckenooghe A, Viviani S, Tornieporth NG, Lang J 2012. Protective efficacy of the recombinant, live-attenuated, CYD tetravalent dengue vaccine in Thai schoolchildren: a randomised, controlled phase $2 \mathrm{~b}$ trial. Lancet 380: 1559-1567.

Siler JF, Hall MW, Hitchens AP 1926. Dengue: its history, epidemiology, mechanism of transmission, etiology, clinical manifestations, immunity and prevention. Philippine J Sci 29: 1-302.

Sim S, Ramirez JL, Dimopoulos G 2012. Dengue virus infection of the Aedes aegypti salivary gland and chemosensory apparatus induces genes that modulate infection and blood-feeding behavior. PLoS Pathog 8: e1002631.

Simmons JS, St John JH, Reynolds FHK 1931. Experimental studies of dengue. Philippine J Sci 44: 1-247.

Weaver SC, Brault AC, Kang W, Holland JJ 1999. Genetic and fitness changes accompanying adaptation of an arbovirus to vertebrate and invertebrate cells. J Virol 73: 4316-4326.

Weaver SC, Lorenz LH, Scott TW 1993. Distribution of western equine encephalomyelitis virus in the alimentary tract of Culex tarsalis (Diptera: Culicidae) following natural and artificial blood meals. J Med Entomol 30: 391-397. 